\title{
DESEMPENHO DAS ATIVIDADES DE VIDA DIÁRIA EM IDOSOS COM ALZHEIMER
}

\author{
Maria Helena Lenardt ${ }^{1}$, Scheilla Cristina da Silva², Márcia Daniele Seima ${ }^{3}$, Mariluci Hautsch Willig ${ }^{4}$, Patrícia
} Aparecida de Oliveira Fuchs ${ }^{5}$

\begin{abstract}
RESUMO: Trata-se de estudo descritivo transversal realizado num Centro de Referência em Doença de Alzheimer do Município de Curitiba, Paraná, cujo objetivo foi avaliar o desempenho das atividades básicas e instrumentais de vida de idosos com Alzheimer. Os dados foram coletados por meio das Escalas de Katz e Lawton e analisados utilizando-se estatística descritiva; a amostra de conveniência foi composta por 55 idosos. Os resultados revelaram que $40 \%$ eram independentes para Atividades Básicas de Vida Diária; 3,6\% totalmente dependentes e 56,4\% parcialmente dependentes. Concernente às Atividades Instrumentais de Vida Diária, 1,8\% dos idosos foram considerados independentes; 27,3\% totalmente dependentes e 70,9\% parcialmente dependentes. Os resultados fornecem subsídios para uma leitura efetiva das reais necessidades destes idosos diante do planejamento de estratégias para protelar a evolução de incapacidades. PALAVRAS-CHAVE: Idoso; Doença de Alzheimer; Enfermagem; Atividades cotidianas.
\end{abstract}

\section{PERFORMANCE OF ACTIVITIES OF DAILY LIVING IN ELDERLY PERSONS WITH ALZHEIMER'S}

\begin{abstract}
A descriptive, transversal study undertaken in a Specialised Centre for Alzheimer's in the municipality of Curitiba, this has as its objective to evaluate the performance of basic and instrumental activities of living among elderly persons with Alzheimer's. The data was collected using the Katz index and the Lawton scale and analysed using descriptive statistics. The convenience sample was composed of 55 elderly patients. The results show that $40 \%$ could carry out basic activities of daily living independently; 3.6\% were totally dependent and $56.4 \%$ were partially dependent. In terms of instrumental activities of daily living, $1.8 \%$ were considered independent; $27.3 \%$ totally dependent; and $70.9 \%$ partially dependent. The results provide support for an effective understanding of these elderly persons' real necessities in terms of planning strategies to slow the progression of their incapacitation.
\end{abstract}

KEYWORDS: Elderly; Alzheimer's; Nursing; activities of daily living.

\section{DESEMPEÑO DE LAS ACTIVIDADES DE VIDA DIARIA EN ANCIANOS CON ALZHEIMER}

RESUMEN: Es un estudio descriptivo transversal realizado en un Centro de Referencia en Enfermedad de Alzheimer del municipio de Curitiba, Paraná, cuyo objetivo fue evaluar el desempeño de las actividades básicas e instrumentales de vida de ancianos con Alzheimer. Los datos fueron recogidos por medio de Escalas de Katz y Lawton y analizados utilizándose estadística descriptiva; la muestra de conveniencia fue compuesta por 55 ancianos. Los resultados revelaron que 40\% eran independientes para Actividades Básicas de Vida Diaria; 3,6\% totalmente dependientes y 56,4\% parcialmente dependientes. Acerca de las Actividades Instrumentales de Vida Diaria, 1,8\% de los ancianos fueron considerados independientes; $27,3 \%$ totalmente dependientes y $70,9 \%$ parcialmente dependientes. Los resultados fornecen subsidios para una lectura efectiva de las reales necesidades de eses ancianos delante del planeamiento de estrategias para aplazar la evolución de incapacidades.

PALABRAS CLAVE: Anciano; Enfermedad de Alzheimer; Enfermería; Actividades cotidianas.

\footnotetext{
${ }^{1}$ Enfermeira. Doutora em Filosofia. Professora do Programa de Pós-Graduação em Enfermagem da Universidade Federal do ParanáPPGENF UFPR. Líder do Grupo Multiprofissional de Pesquisa sobre Idosos-GMPI UFPR.

${ }^{2}$ Enfermeira. Mestre em Ciências da Saúde.

${ }^{3}$ Enfermeira. Doutoranda no PPGENF UFPR. Membro do GMPI.

${ }^{4}$ Enfermeira do Hospital de Clínicas-HC UFPR. Doutoranda no PPGENF UFPR. Membro do GMPI UFPR.

${ }^{5}$ Enfermeira Assistencial do Pronto-Socorro do Hospital Universitário Cajuru-Pontifícia Universidade Católica do Paraná.
}

Autor correspondente:

Márcia Daniele Seima

Universidade Federal do Paraná

R. Ana Berta Roskamp, 284 - 81530-250 - Curitiba-PR-Brasil

Recebido: $15 / 12 / 10$

E-mail: marciaseima@gmail.com

Aprovado: 15/02/11 


\section{INTRODUÇÃO}

O processo de envelhecimento humano traz modificações na qualidade de vida dos idosos, principalmente na capacidade funcional entendida como a habilidade do indivíduo em desempenhar, de forma independente, as atividades ou tarefas cotidianas, identificadas como essenciais para a manutenção do seu bem-estar ${ }^{(1)}$.

A habilidade para realizar as tarefas cotidianas envolve responsabilidade a ser compartilhada entre idosos, cuidadores e profissionais, por meio de cuidados coordenados e contínuos que proporcionem as condições básicas para um desempenho satisfatório.

$\mathrm{O}$ risco para a incapacidade funcional se agrava quando o idoso é acometido por demência, particularmente a Doença de Alzheimer (DA). O principal fator de risco para essa doença é a idade. Sua incidência dobra a cada cinco anos após os 65 anos de vida ${ }^{(2)}$. Dados sobre indivíduos centenários mostram que a DA não é, necessariamente, o resultado do envelhecimento ${ }^{(3)}$, no entanto, as chances de receber o diagnóstico de DA após os 85 anos de idade são de uma em cada três idosos ${ }^{(4)}$.

A DA emerge com alteração neurológica, de processo degenerativo, causada pela atrofia progressiva, com perdas de células do córtex cerebral, hipocampo, estruturas subcorticais e uma deficiência múltipla de neurotransmissores ${ }^{(5)}$. Conforme a patologia evolui, aumentam os sinais e sintomas da demência, que culminam na dependência do idoso portador de Alzheimer. Na sua fase inicial, o comprometimento da memória é o sintoma mais evidente, evoluindo para a fase moderada, caracterizada pela dificuldade de aprendizagem, afasia, apraxia, agnosia e alterações visuoespaciais. Nesta fase, os idosos apresentam maiores dificuldades na realização das atividades cotidianas, ocasionando, não apenas um declínio cognitivo, mas também funcional. Na fase final da doença, os idosos tornam-se totalmente dependentes de cuidados $^{(6)}$.

As atividades cotidianas são caracterizadas pelas atividades básicas de vida diária (ABVD), as quais englobam a mobilidade básica (transferência, deambulação) e o cuidado de si (alimentação, higiene pessoal, vestir-se). Já as atividades instrumentais de vida diária (AIVD) se referem a um conjunto vasto de tarefas comuns que um indivíduo deve ser capaz de executar para manter sua independência, autonomia e relacionamento social, tais como: preparar alimentos, administrar o dinheiro, utilizar transporte e se medicar.
Para avaliar o grau de dependência do idoso em executar atividades que permitam cuidar de si e viver independentemente, pode ser utilizado um conjunto de dados clínicos, testes e escalas. Para este estudo optou-se pelas escalas de $\mathrm{Katz}^{(7)}$ para as ABVD e de Lawton ${ }^{(8)}$ para as AIVD, por serem breves, simples e de fácil aplicação ${ }^{(9)}$, além de poderem ser aplicadas tanto na área hospitalar e ambulatorial quanto em unidades de saúde. A avaliação breve pode auxiliar no rastreamento dos idosos com risco de adoecer gravemente, com risco de hospitalização e aqueles susceptíveis de perder a capacidade de desempenhar as atividades de vida diária ${ }^{(10)}$.

O rastreamento precoce de alterações cognitivas e funcionais se faz necessário, principalmente, para os idosos portadores de DA visto que, já nas fases iniciais e moderadas da doença, estes podem apresentar diferentes graus de dependências. Em pesquisa realizada na Bélgica, com 1289 pacientes com DA em estágio leve e moderado, foi constatado que $69 \%$ já necessitavam de mais de 12 horas de supervisão por dia, o que demonstra que, no início da evolução da doença, os pacientes apresentam-se bastante dependentes e com déficit da capacidade funcional ${ }^{(11)}$.

O mesmo resultado foi encontrado em estudo nacional recente, o qual concluiu que os sintomas da doença afetam a qualidade de vida dos idosos já nas fases leves e moderadas ${ }^{(12)}$.

Diagnosticar precocemente os idosos com DA que apresentam riscos para perda da capacidade de desempenhar as atividades de vida diária representa o primeiro estágio do processo para protelar as perdas manifestadas no transcorrer da doença. Ele indica um cuidado contínuo que se torna um imperativo no processo de viver diário do idoso com Alzheimer.

A avaliação do desempenho das atividades de vida diária viabiliza propostas de reabilitação eficazes e orienta as ações de cuidado dos profissionais da saúde e dos cuidadores dos idosos com DA, cujo principal objetivo é protelar os agravos da doença e favorecer melhor qualidade de vida para o portador e para o cuidador $^{(13)}$. Diante do exposto o estudo teve como objetivo avaliar o desempenho das Atividades Básicas e Instrumentais de Vida Diária (ABVD e AIVD) de idosos com Alzheimer.

\section{METODOLOGIA}

Trata-se de estudo descritivo transversal, realizado em um Centro de Referência em Doença de 
Alzheimer - Unidade de Atenção ao Idoso, na cidade de Curitiba, Estado do Paraná, no período de janeiro a fevereiro de 2007. Os usuários da unidade que apresentavam demência do tipo Alzheimer constituíramse num quantitativo de 1.073 idosos, até o mês de abril de 2007. A seleção dos idosos ocorreu por meio de critérios de inclusão e obteve-se a amostra de conveniência de 55 idosos. Foram critérios de inclusão: apresentar diagnóstico médico de DA, encontrar-se em estágio inicial ou moderado da doença, ter cuidador familiar, estar em tratamento na unidade de saúde, e aceitar participar do estudo por livre e espontânea vontade.

Para a avaliação das atividades cotidianas a coleta de dados ocorreu junto aos pacientes com DA, aplicando-se as escalas autorreferidas de ABVD, conforme $\mathrm{Katz}^{(7)}$ e AIVD, indicadas por Lawton ${ }^{(8)}$.

A escala de $\mathrm{Katz}^{(7)}$ avalia seis atividades (tomar banho, vestir-se, usar o banheiro, transferência, continência urinária/fecal, e alimentar-se) e o resultado pode variar entre zero e seis pontos, sendo que o valor mínimo (zero) significa que o indivíduo é dependente para as ABVD e o máximo (seis) que é independente. A escala de Lawton ${ }^{(8)}$ avalia oito atividades (usar o telefone, fazer compras, preparar a refeição, fazer faxina, lavar e passar roupa, usar meio de transporte, tomar medicações e realizar o controle financeiro). O escore pode variar entre 9 e 27 pontos, sendo que o valor máximo significa que o indivíduo é independente para as AIVD.

Os escores foram atribuídos de acordo com a pontuação sugerida pelos autores ${ }^{(7-8)}$ dessas escalas e tratados por meio de estatística descritiva, com dados de frequência simples e percentual, utilizando-se o programa Epi Info 6.0. Os registros em prontuários foram fontes secundárias de coletas de dados, que permitiram selecionar os idosos de acordo com algum dos critérios de inclusão.

A análise da associação entre as ABVD, mais especificamente, as atividades de tomar banho, alimentar-se e transferência, com a atividade de continência, e a relação das AIVDs - fazer compras e tomar medicação - com a utilização do meio de transporte, e entre uma ABVD - alimentar-se, e uma AIVD - cozinhar, foram apresentados em tabelas e dados de estatística descritiva.

O projeto foi analisado e aprovado pelo Comitê Setorial de Ética do Setor de Ciências da Saúde da Universidade Federal do Paraná - n. 0008.0.085.0910, e pela Comissão de Ética em Saúde da Secretaria
Municipal de Saúde de Curitiba (parecer favorável em 24 de janeiro de 2007). Em todas as etapas da pesquisa foram consideradas e cumpridas as bases da Resolução 196/96. Os idosos que manifestaram concordância em participar assinaram o Termo de Consentimento Livre e Esclarecido.

\section{RESULTADOS}

A idade dos participantes do estudo variou de 60 a 89 anos e teve média de 76,24 anos, com um desvio padrão de 7,5 anos. Constatou-se que dos 55 idosos com DA $25,45 \%$ são do sexo masculino e $74,55 \%$ do sexo feminino.

$O$ resultado referente à avaliação das ABVD variou entre 0 e 6 pontos, com média de 4,33 e desvio padrão de 1,785 , sendo que $3,6 \%$ dos idosos apresentaram dependência total; enquanto $40 \%$ dos idosos atingiram a pontuação máxima e foram considerados independentes. Outros 56,4\% foram incluídos na categoria de semi-dependentes, os quais apresentaram comprometimento de uma das funções influenciadas pela cultura ou aprendizado, como tomar banho, vestir-se ou usar o banheiro ou de uma das funções vegetativas simples (transferência e /ou continência).

Entre as ABVD, as que mais se destacaram como realizáveis foram: alimentar-se (52), transferência (48) e continência (45) (Tabela 1).

Tabela 1 - Atividades básicas de vida diária segundo Escala de Katz referente à continência associados a alimentar-se, transferência e tomar banho. Curitiba, 2007

\begin{tabular}{lcccc}
\hline & \multicolumn{3}{c}{ Continência } & Total \\
\hline \multirow{4}{*}{ Alimentar-se } & & Não & Sim & \\
& Não & 2 & 1 & 3 \\
& Sim & 8 & 44 & 52 \\
& Total & 10 & 45 & 55 \\
Transferência & Não & 5 & 2 & 7 \\
& Sim & 5 & 43 & 48 \\
& Total & 10 & 45 & 55 \\
& Não & 10 & 16 & 26 \\
& Sim & 0 & 29 & 29 \\
& Total & 10 & 45 & 55 \\
\hline \multirow{7}{*}{ Tomar banho }
\end{tabular}

Ao associar as variáveis continência e alimentação, verificou-se que 44 idosos são independentes para essas duas atividades; para continência e transferência 
observou-se que 43 idosos são independentes e, quando comparadas continência e banho, constatou-se que 29 são capazes de realizar as duas ABVD.

$\mathrm{Na}$ tabela 2 visualiza-se a relação entre as faixas etárias e as ABVD. "Alimentar-se" obteve o maior desempenho das atividades realizáveis, equivalente a 52 $(94,5 \%)$ do total. Destes, 24 (43,6\%) dos participantes tinham entre 70 e 79 anos, 8 (14,5\%) deles estavam na faixa etária dos 60 a 69 anos e todos os $20(36,4 \%)$ idosos com 80 anos ou mais executavam esta tarefa. A atividade que obteve o segundo melhor desempenho foi sair da poltrona, com um total de $48(87,3 \%)$ usuários. Ao associar a variável faixa etária, $23(41,8 \%)$ idosos considerados independentes para a atividade de transferência se encontravam entre 70 a 79 anos, 19 (34,5\%) deles com 80 anos ou mais e $6(10,9 \%)$ entre 60 e 69 anos.

Tabela 2 - Relação das frequências entre a faixa etária e as atividades básicas de vida diária segundo Escala de Katz. Curitiba, 2007

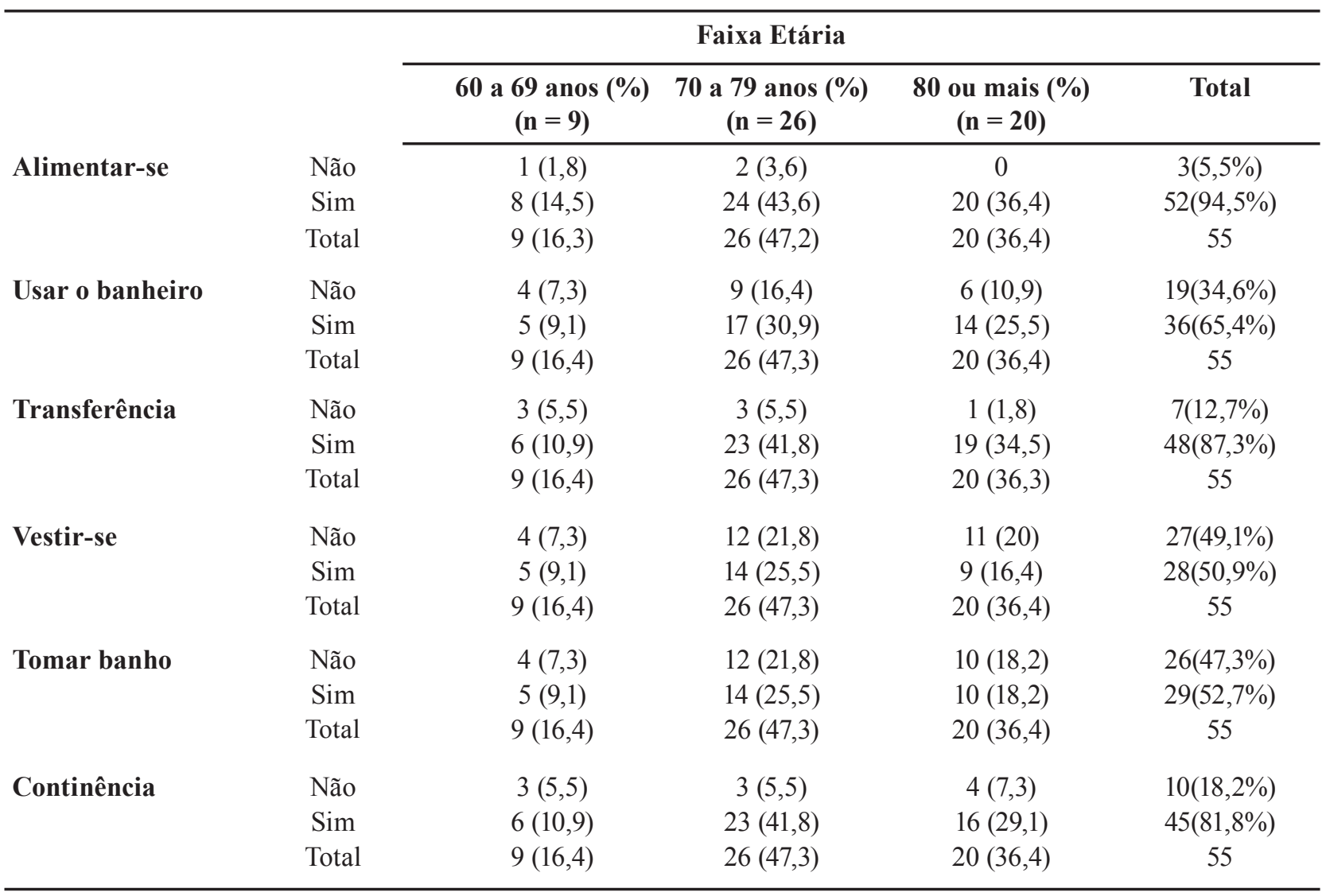

Os idosos apresentaram um escore que variou entre 9 e 27 e desvio padrão de 4,48 pontos, apenas um idoso era independente para as AIVD, isto é, atingiu o escore máximo; apresentaram dependência total para as AIVD 15 idosos, e 39 foram classificados como semi-dependentes.

Identificou-se que em AIVD 87,3\% dos idosos apresentaram dificuldades para administrar o dinheiro, $87,3 \%$ não utilizamvam transporte, $78,2 \%$ deles não faziam compras e $74,6 \%$ não conseguiam tomar medicamentos sem auxílio.

Observa-se, na tabela 3, que ao comparar a capacidade de realização de duas AIVD, como fazer com- pras e usar o transporte, constatou-se que 41 pacientes não realizavam essas atividades e 5 desempenhavam as duas atividades instrumentais. Ao relacionar a utilização de transportes e a administração do dinheiro verificou-se que 43 usuários não realizavam estas atividades e que apenas 2 as realizavam sem auxílio, e 4 conseguiam utilizar o transporte sem auxílio, porém não administravam o dinheiro.

Ao relacionar as frequências entre as faixas etárias e as AIVD, constatou-se que 29 (52,8\%) idosos eram capazes de desenvolver tarefas rotineiras, desses, 11 (20\%) possuíam 80 anos ou mais, $14(25,5 \%)$ tinham entre 70 e 79 anos e $4(7,3 \%)$ entre 60 e 69 anos. 
Tabela 3 - Relação de duas atividades instrumentais de vida diária - fazer compras, administrar dinheiro - com usar transporte, segundo Escala de Lawton. Curitiba, 2007

\begin{tabular}{lccccc}
\hline & & & Usar transporte & & Total \\
\hline \multirow{3}{*}{ Fazer compras } & & Não consegue & Com ajuda & Sim & \\
& Não consegue & 41 & 1 & 1 & 43 \\
& Com ajuda & 4 & 0 & 0 & 4 \\
& Sim & 3 & 0 & 5 & 8 \\
\multirow{3}{*}{ Administrar o dinheiro } & Total & 48 & 1 & 6 & 55 \\
& & 43 & 1 & 4 & 48 \\
& Não consegue & 2 & 0 & 0 & 2 \\
& Com ajuda & 3 & 0 & 2 & 5 \\
& Sim & 48 & 1 & 6 & 55
\end{tabular}

O uso de transporte e administração do dinheiro foram as atividades com maior nível de dependência, somando $48(87,2 \%)$ do total de idosos (Tabela 4). Ao categorizar a atividade administrar o dinheiro, observou-se que $23(41,8 \%$ ) idosos que não desempenhavam esta função possuíam entre 70 e 79 anos, $17(30,9 \%)$ com 80 ou mais anos e $8(14,5 \%)$ na faixa etária de 60 a 69 anos. Quanto à utilização de transporte, o número de idosos dependentes com 80 anos ou mais aumentou para $18(32,7 \%)$, já os que apresentavam entre 70 e 79 anos foram 22 (40\%), e entre 60 e 69 anos foram somados $8(14,5 \%)$.

Ao relacionar ABVD com as AIVD, como cozinhar e alimentar-se, observou-se que 3 idosos não realizavam essas atividades de forma independente, 13 eram capazes de alimentar-se e capazes de cozinhar, e 37 alimentavam-se de forma independente, porém eram incapazes de cozinhar.

Vale ressaltar que, neste estudo, a maior parte dos idosos encontrava-se na categoria de parcialmente dependentes, tanto para as ABVD $(56,4 \%)$ quanto para as $\operatorname{AIVD}(70,9 \%)$.

Tabela 4 - Relação das frequências entre a faixa etária e as atividades instrumentais de vida diária segundo Escala de Lawton. Curitiba, 2007

Faixa Etária

\begin{tabular}{|c|c|c|c|c|c|}
\hline & & $\begin{array}{c}60 \text { a } 69 \text { anos } \\
\text { N (\%) }\end{array}$ & $\begin{array}{c}70 \text { a } 79 \text { anos } \\
\text { N (\%) }\end{array}$ & $\begin{array}{c}80 \text { ou mais } \\
\text { N }(\%)\end{array}$ & $\begin{array}{l}\text { Total } \\
\text { N (\%) }\end{array}$ \\
\hline \multirow[t]{4}{*}{ Administrar o dinheiro } & Não consegue & $8(14,5)$ & $23(41,8)$ & $17(30,9)$ & $48(87,2)$ \\
\hline & Com ajuda & 0 & $2(3,6)$ & 0 & $2(3,6)$ \\
\hline & $\operatorname{Sim}$ & $1(1,8)$ & $1(1,8)$ & $3(5,5)$ & $5(9,1)$ \\
\hline & Total & $9(16,3)$ & $26(47,2)$ & $20(36,4)$ & 55 \\
\hline \multirow[t]{4}{*}{ Lavar e passar roupa } & Não consegue & $4(7,3)$ & $20(36,4)$ & $15(27,3)$ & $39(71)$ \\
\hline & Com ajuda & $2(3,6)$ & 0 & $1(1,8)$ & $3(5,5)$ \\
\hline & Sim & $3(5,5)$ & $6(10,9)$ & $4(7,3)$ & $13(23,7)$ \\
\hline & Total & $9(16,4)$ & $26(47,3)$ & $20(36,4)$ & 55 \\
\hline \multirow[t]{4}{*}{ Fazer compras } & Não consegue & $7(12,7)$ & $19(34,5)$ & $17(30,9)$ & $43(78,1)$ \\
\hline & Com ajuda & $1(1,8)$ & $3(5,5)$ & 0 & $4(7,3)$ \\
\hline & Sim & $1(1,8)$ & $4(7,3)$ & $3(5,5)$ & $8(14,5)$ \\
\hline & Total & $9(16,3)$ & $26(47,3)$ & $20(36,4)$ & 55 \\
\hline
\end{tabular}




\begin{tabular}{|c|c|c|c|c|c|}
\hline & & \multicolumn{4}{|c|}{ Faixa Etária } \\
\hline & & $\begin{array}{c}60 \text { a } 69 \text { anos } \\
\times(\%)\end{array}$ & $\begin{array}{c}70 \text { a } 79 \text { anos } \\
N(\%)\end{array}$ & $\begin{array}{c}80 \text { ou mais } \\
\mathrm{N}(\%)\end{array}$ & $\begin{array}{l}\text { Total } \\
\text { N (\%) }\end{array}$ \\
\hline \multirow{4}{*}{ Tomar remédios } & Não consegue & $7(12,7)$ & $19(34,5)$ & $15(27,3)$ & $41(74,5)$ \\
\hline & Com ajuda & $2(3,6)$ & $5(9,1)$ & 0 & $7(12,7)$ \\
\hline & Sim & 0 & $2(3,6)$ & $5(9,1)$ & $7(12,7)$ \\
\hline & Total & $9(16,3)$ & $26(47,2)$ & $20(36,4)$ & 55 \\
\hline \multirow[t]{4}{*}{ Cozinhar } & Não consegue & $5(9,1)$ & $20(36,4)$ & $15(27,3)$ & $40(72,8)$ \\
\hline & Com ajuda & $2(3,6)$ & 0 & 0 & $2(3,6)$ \\
\hline & Sim & $2(3,6)$ & $6(10,9)$ & $5(9,1)$ & $13(23,7)$ \\
\hline & Total & $9(16,3)$ & $26(47,3)$ & $20(36,4)$ & 55 \\
\hline \multirow[t]{4}{*}{ Fazer faxina } & Não consegue & $6(10,9)$ & $16(29,1)$ & $14(25,5)$ & $36(65,5)$ \\
\hline & Com ajuda & $1(1,8)$ & 0 & $1(1,8)$ & $2(3,6)$ \\
\hline & Sim & $2(3,6)$ & $10(18,2)$ & $5(9,1)$ & $17(30,9)$ \\
\hline & Total & $9(16,3)$ & $26(47,3)$ & $20(36,4)$ & 55 \\
\hline \multirow[t]{4}{*}{ Fazer tarefas rotineiras } & Não consegue & $2(3,6)$ & $9(16,4)$ & $7(12,7)$ & $18(32,7)$ \\
\hline & Com ajuda & $3(5,5)$ & $3(5,5)$ & $2(3,6)$ & $8(14,6)$ \\
\hline & Sim & $4(7,3)$ & $14(25,5)$ & $11(20)$ & $29(52,8)$ \\
\hline & Total & $9(16,4)$ & $26(47,4)$ & $20(36,3)$ & \\
\hline \multirow[t]{4}{*}{ Usar transporte } & Não Consegue & $8(14,5)$ & $22(40)$ & $18(32,7)$ & $48(87,2)$ \\
\hline & Com ajuda & 0 & 0 & $1(1,8)$ & $1(1,8)$ \\
\hline & Sim & $1(1,8)$ & $4(7,3)$ & $1(1,8)$ & $6(10,9)$ \\
\hline & Total & $9(16,3)$ & $26(47,3)$ & $20(36,3)$ & 55 \\
\hline \multirow[t]{4}{*}{ Usar o telefone } & Não consegue & $6(10,9)$ & $13(23,6)$ & $12(21,8)$ & $31(56,3)$ \\
\hline & Com ajuda & $3(5,5)$ & $7(12,7)$ & $2(3,6)$ & $12(21,8)$ \\
\hline & Sim & 0 & $6(10,9)$ & $6(10,9)$ & $12(21,8)$ \\
\hline & Total & $9(16,4)$ & $26(47,2)$ & $20(36,3)$ & 55 \\
\hline
\end{tabular}

Tabela 5 - Relação de atividade básica de vida diária e atividades instrumentais de vida diária - cozinhar e alimentar-se - segundo a Escala de Lawton. Curitiba, 2007

\section{DISCUSSÃO}

A predominância de idosos do sexo feminino portadores da DA é apontada também em estudo ${ }^{(13)}$, envolvendo cerca de 36 idosos, onde $66,7 \%$ eram do sexo feminino, dados estes que se assemelham aos achados nesta pesquisa.

A porcentagem do nível de independência para a realização de ABVD (40\%) contrapõe-seaos do estudo realizado em Porto Alegre/RS que avaliou 36 idosos com DA e apontou que $22,2 \%$ apresentavam independência para a realização das ABVD, outros $22,2 \%$ dependência parcial, e $55,6 \%$ dependência total. Salienta-se que autores não especificaram em qual fase da doença os idosos se encontravam ${ }^{(14)}$.

Já os resultados do presente estudo aproximamse aos encontrados em pesquisa realizada em Natal/ RN, no ano de 2009, com avaliação de 15 idosos com DA. Verificou-se que o escore médio foi de 10, havendo um idoso que apresentou capacidade de realizar todas as atividades sem ajuda e outro com a capacidade de realizar todas com ajuda ${ }^{(15)}$.

Ainda, identificou-se pontuação média de 11,7 idosos classificados como semidependentes, sendo que três dos idosos apresentaram dependência total ${ }^{(15)}$. Os resultados corroboram com os encontrados neste estudo, no qual a maioria dos idosos (70,9\%) é classificada como semidependentes.

De acordo com a literatura, há uma hierarquia no declínio funcional. Os indivíduos perdem, inicialmente, o desempenho das tarefas mais com- 
plexas (AIVD) e aprendidas culturalmente, e por último, deixam de realizar as ABVD. Refletindo, desta maneira, um grau mais alto de dependência ${ }^{(1)}$.

Os dados encontrados neste estudo em relação às AIVD também confirma os achados da pesquisa realizada no Rio de Janeiro: do total de 16 idosos, 3 indivíduos necessitavam de ajuda em duas atividades e 13 eram dependentes parciais ou totais nas atividades de preparo da alimentação, administração de medicamentos, cuidados com a vestimenta, auxílio para a movimentação, administração de alimentos, usar o toalete e realizar curativos ${ }^{(16)}$.

Em pesquisa desenvolvida em um serviço ambulatorial de geriatria do Município de São Paulo, com 100 idosos, verificou-se que as limitações funcionais são mais frequentes em indivíduos mais velhos, e que o risco de incapacidade funcional aumenta consideravelmente com o passar dos anos ${ }^{(17)}$, o que também confirmou-se neste estudo.

Conforme a DA progride, maiores são os comprometimentos funcionais para o desempenho das ABVD e AIVD. Este fato corrobora com os achados de pesquisa realizada com 2.143 idosos, na qual a incidência de dificuldades entre os idosos com declínio para o desempenho das AIVD mostrouse maior que das ABVD. Nas AIVD, apenas 2,4\% mencionaram não possuir dificuldade alguma, $11,4 \%$ indicaram uma ou duas dificuldades e a maioria $(86,2 \%)$ apresentou três ou mais dificuldades. Já nas ABVD, 81,4\% mantêm-se independente para todas as atividades ${ }^{(18)}$.

Contatou-se, no presente trabalho que a maioria dos idosos foi classificada como parcialmente dependente, tanto para as ABVD, quanto para as AIVD.Esses dados são preocupantes, pois os idosos apresentam grande potencial para tornar-se dependentes, como observado na pesquisa realizada no Programa de Geriatria e Gerontologia da Universidade Federal Fluminense, com 11 idosos demenciados entre agosto de 2006 a julho de 2007.

Os dados foram coletados trimestralmente e evidenciou-se que na primeira etapa todos os idosos eram semidependentes para as AIVD, dentre esses, quatro idosos diminuíram a pontuação nas avaliações seguintes. Quanto às ABVD, três idosos apresentavam-se independentes para a realização das atividades e outros oito eram semidependentes; destes, dois evoluíram para escores mais baixos na segunda avaliação e mais quatro na terceira avaliação ${ }^{(19)}$.

\section{CONCLUSÕES}

Os resultados do presente estudo mostraram que grande parcela dos idosos (40\%) com demência do tipo Alzheimer preservava uma ou mais ABVD no estágio inicial e moderado da doença, o que indica que essas atividades precisam ser valorizadas pelos cuidadores e profissionais, com o intuito de promover a autonomia e independência pelo maior tempo possível.

Concernente às AIVD, os escores revelaram que o idoso possui maior dificuldade no cuidado com dinheiro e em utilizar transporte, situações fundamentais para o convívio social. Estes fatores conduzem o idoso ao isolamento social e, consequentemente, à permanência em período integral no domicílio. A necessidade de um cuidador torna-se fundamental. O idoso portador da DA consegue cuidar de si no ambiente domiciliar (ABVD), mas não possui independência no ambiente externo (AIVD).

Ao realizar levantamento da literatura para discussão dos achados nesta pesquisa, evidenciou-se, o déficit de estudos que abordem, de modo específico, a fase da DA em que se encontra o idoso. Esse dado torna problemático o processo de determinação, comparação e interpretação da capacidade funcional dos idosos dentre os estudos realizados.

Faz-se necessário o desenvolvimento de pesquisas que avaliem o desempenho da capacidade funcional em segmento populacional específico como: de idosos jovens e de idosos muito idosos. Os estudos disponíveis abordam o assunto, porém, de modo generalizado.

Acredita-se que esta pesquisa traz um sentido inovador ao mostrar que os idosos com Alzheimer, já nas fases iniciais e moderadas, apresentam forte potencial para tornar-se dependentes, tanto nas atividades instrumentais quanto nas básicas.

É relevante para a Enfermagem Gerontológica identificar o desempenho de idosos com DA frente às atividades cotidianas (livre de sujeição de determinada escala), visto que os resultados oferecem subsídios para repensar as estratégias de intervenção utilizadas nos serviços de Atenção à Saúde, com o intento de protelar os processos de perdas provocados pela doença.

Considera-se imprescindível, e um ganho significativo para o acompanhamento do idoso com DA, a implementação das atividades de vida diária, 
como instrumento básico de avaliação.

Salienta-se que é também preciso avançar na construção do processo de orientação da atenção ao idoso com Alzheimer. Corroboram com esse desejo os profissionais de enfermagem envolvidos com o cuidado de idosos, pois constatam diariamente a necessidade da avaliação sistemática, segundo os padrões preconizados pela Gerontologia, e que possibilitam ter em mãos dados concretos e a real situação e evolução da doença.

\section{REFERÊNCIAS}

1. Moraes EN, Moraes FL, Keller A, Ribeiro MTF. Avaliação clínico-funcional do idoso. In: Moraes EN. Princípios básicos de geriatria e gerontologia. Belo Horizonte (MG): Coopmed; 2008. p. 63-83.

2. Hirtz D, Thurman DJ, Gwinn-Hardy K, Mohamed M, Chaudhuri AL, Zalutsky R. How common are the "common" neurologic disorders? Neurology 2007;68:326-37.

3. Dunnen WF, Brouwer WH, Bijlard E. No disease in the brain of a11 5-year-old woman. Neurobiol Aging. 2008;29(8):1127-32.

4. Querfurth HW, LaFerla FM. Mechanisms of disease. The New England Journal of Medicine. 2010;362(4):329-44.

5. Antczak SE, Berger N, Conroy WT, Craig L, Durston S, Hanes DA, et al. Fisiopatologia básica. Rio de Janeiro: Guanabara Koogan; 2005.

6. Machado JCB. Doença de Alzheimer. In: Freitas EV, Py L, Cançado FAX, Gorzoni ML. Tratado de geriatria e gerontologia. $2^{\mathrm{a}}$ ed. Rio de Janeiro: Guanabara Koogan; 2002.

7. Katz S, Ford AB, Moskowitz RW, Jackson BA, Jaffe MW. Studies of illness in de aged. The index of ADL: a standardized measure of biologycal and psychosocial function. JAMA. 1963;185:914-9.

8. Lawton MP, Brody EM. Assessment of older people: self-maintaining and instrumental activities of daily living. Gerontologist. 1969;9(3):179-86.

9. Costa EFA, Monego ET. Avaliação geriátrica ampla (AGA). Rev UFG [Internet]. 2003 Dez; 5(2) [acesso em 07 jun 2010] Disponível: http://www.proec.ufg. br/revista_ufg/idoso/aga.html
10. Veras RP, Caldas CP, Coelho FD, Sanchez MA. Promovendo a saúde e prevenindo a dependência: identificando indicadores de fragilidade em idosos independentes. Rev Bras Geriatr Gerontol. 2007;10(3):355-70.

11. Caro J, Ward A, Ishak K, Migliaccio - Walle K, Getsios D, Papadopulos G et al. To what degree does cognitive impairment in Alzheimer's disease predict dependence of patients on caregivers? BMC Neurology [Internet]. 2002;2:6 [acesso em 07 jun 2010]. Disponível: http://www.biomedcentral. com/1471-2377/2/6.

12. Novelli MMPC, Caramelli P. The influence of neuropsychiatric and functional changes on quality of life in Alzheimer's disease. Dement Neuropsychol. 2010;4(1):47-53.

13. Medeiros ME, Guerra RO. Tradução, adaptação e análises das propriedades psicométricas do activities of daily living questionnaire (ADLQ) para avaliação funcional de pacientes com a doença de Alzheimer. Rev Bras Fisioter. 2009;13(3):257-66.

14. Luzardo AR, Gorini MIPC, Silva APSS. Características de idosos com doença de Alzheimer e seus cuidadores: uma série de casos em um serviço de neurogeriatria. Texto \& Contexto Enferm. 2006;15(4):587-94.

15. Lemos CA, Rocha EA, Papaleo SS, Maia EMC, Pires IAH. Cuidadores de idosos com doença de Alzheimer: capacidade funcional do idoso "versus" avaliação da sobrecarga do cuidado. In: III Congresso Ibero-Americano de Psicogerontologia: subjetividade, cultura e poder; 2009 Nov. p.3-5; São Paulo: PUCSP; 2009.

16. Santana RF, Santos I, Caldas CP. Cuidando de idosos com demência: um estudo a partir da prática ambulatorial de enfermagem. Rev Bras Enferm. 2005;58(1):44-8.

17. Minosso JSM, Amendola F, Alvarenga MRM, Oliveira MAC. Prevalência de incapacidade funcional e dependência em idosos atendidos em um centro de saúde-escola da Universidade de São Paulo. Cogitare Enferm. 2010;15(1):12-8

18. Oliveira SFD, Duarte YAO, Lebrão ML, Laurenti R. Demanda referida e auxílio recebido por idosos com declínio cognitivo no município de São Paulo. Saúde Soc. 2007;16(1):1-13. 
19. Souza PA, Bastos RCS, Santana RF, Sá SPC, Cassino KM. Oficinas de estimulação cognitiva para idosos com demência: uma estratégia de cuidado na enfermagem gerontológica. Rev Gaúch Enferm. 2008;29(4):588-95. 